\title{
Diagnóstico convectivo operativo a partir del modelo HRES-IFS
}

\author{
Jesús Riesco Martín'1 (jriescom@aemet.es) \\ Carlos Jiménez Alonso ${ }^{1}$ (cjimenza@aemet.es) \\ Carlos Perea Hitos ${ }^{1}$ (cpereah@aemet.es)
}

${ }^{1}$ AEMET / Delegación Territorial en Andalucía / Centro Meteorológico de Málaga

\begin{abstract}
RESUMEN
Se ha desarrollado una aplicación interna en AEMET que trata de recopilar un amplio conjunto de campos y parámetros que han demostrado su utilidad real o potencial en el diagnóstico convectivo y de los fenómenos asociados. A tal efecto está disponible una página web operativa en el Sistema Nacional de Predicción, con URL: http://conveclx.aemet.es/ usermal/bucles conveccion/.

Se trata de un diagnóstico convectivo realizado a partir de un modelo hidrostático como es el modelo operativo HRES-IFS del ECMWF, con dos pasadas al día (0 y 12 UTC) y alcances hasta H+72 para esta aplicación.

Los productos generados para el diagnóstico convectivo se han agrupado en ocho bloques principales en función de su propia naturaleza o a partir de los fenómenos generados en superficie. Estos grupos son los siguientes: convección (en general), forzamiento dinámico, campos e índices termodinámicos, cizalladura-organización, granizo y rayos, supercélulas y tornados, vientos convectivos severos no tornádicos y precipitaciones intensas.
\end{abstract}

PALABRAS CLAVE: convección; fenómenos convectivos; modelo hidrostático; HRES-IFS; diagnóstico convectivo; Sistema Nacional de Predicción.

\section{INTRODUCCIÓN}

En la máquina «kumori.aemet.es» del Centro Meteorológico de Málaga, se dispone de una aplicación web en AEMET de uso operativo interno en el Sistema Nacional de Predicción, que trata de recopilar un conjunto de campos y parámetros que han demostrado su utilidad real o potencial, en el diagnóstico de la convección y de fenómenos adversos asociados. Todo ello a partir de modelos hidrostáticos como básicamente el HRES-IFS del ECMWF, ya que el Hirlam en 2018 está en fase de desaparición como modelo operativo en AEMET. Todos estos campos y parámetros están una máquina de Servicios Centrales: intranet http://conveclx.aemet.es/ usermal/bucles_conveccion/y en la página del Centro Meteorológico de Málaga, http://kumori.aemet.es/ bucles_conveccion, con un duplicado en http://kaminari.aemet.es/bucles_conveccion/.

Las salidas se obtienen a partir de las dos pasadas de 0 y 12 UTC del modelo determinista HRES-IFS del Centro Europeo de Predicción a Medio Plazo, según las presentaciones internas en McIdas denominadas (ECMP/ECMC), según sea la representativa de un entorno de Península-Baleares o de Canarias. El alcance de la predicción es de 3 en 3 horas hasta $\mathrm{H}+72$ y de 6 horas entre $\mathrm{H}+72$ y $\mathrm{H}+120$. En la versión inicial, ya en desuso, se contemplaban también las salidas del modelo Hirlam. 
Hay un selector de fechas que permite reproducir las salidas de pasadas anteriores, hasta una antigüedad de unos 5 meses. También la web dispone de una ayuda donde se incluyen a su vez novedades y referencias básicas. La generación de productos se hace a partir de McIdas, donde se han ingestado los ficheros que contienen las predicciones de los campos básicos y derivados, y a su vez se realiza un postproceso especial que permite disponer de un amplio conjunto de campos y parámetros de interés para el predictor.

Los productos generados se han agrupado en ocho grupos principales en función de su propia naturaleza o a partir de los fenómenos generados en superficie. El aspecto de la aplicación web se presenta en la figura 1.

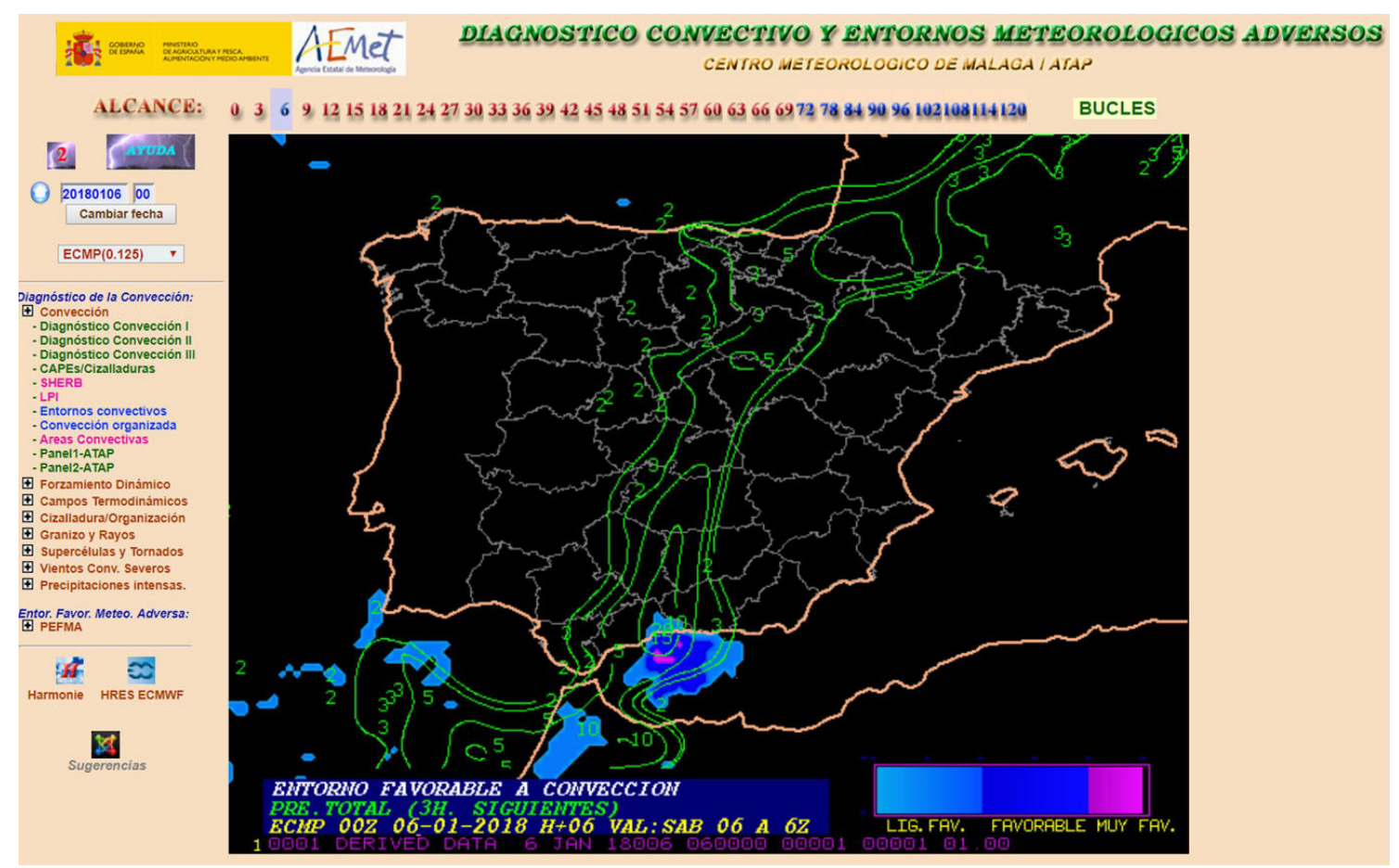

Figura 1. Vista general de la apariencia de la aplicación web.

En la parte superior aparecen los alcances de predicción y a la izquierda los ocho grupos principales con los distintos campos y parámetros, así como acceso a otros productos relacionados con fenomenología adversa en general (PEFMA, acrónimo de «predicción de entornos favorables para meteorología adversa»), en la que los campos generados se refieren a la cuantificación empírica de la predisposición del entorno atmosférico para que se puedan producir distintos tipos de adversidad meteorológica (en gran parte en relación con la convección). El resto del área se reserva para la visualización de campos, y puede estar dividida en uno o varios paneles. El nombre de los campos/parámetros está codificado en tres colores en virtud de su posible uso operativo: verde (índice totalmente operativo), magenta (índice experimental que debe usarse con precaución) y azul (campo del PEFMA).

\section{OBJETIVO, INTERPRETACIÓN Y ESTRUCTURA DE LA PÁGINA}

El objetivo último de la aplicación es proveer al predictor una herramienta más, que especialmente permita focalizar su atención a partir de campos básicos y derivados calculados automáticamente en las zonas especialmente destacadas, las cuales pueden indicar la existencia de entornos favorables o ingredientes disparadores de la convección, así como la aparición de determinados fenómenos convectivos. Es, por tanto, una fuente más de consulta que trata de ayudar al predictor en el diagnóstico convectivo y en la toma posterior de decisiones operativas. Por supuesto, la consideración de distintas herramientas y productos y las habilidades personales del predictor en función de su experiencia y conocimientos, llevará a una mejor predisposición de 
cara al acierto en el pronóstico de situaciones de tipo convectivo, sobre todo en las potencialmente adversas que conducen a la generación de avisos (teniendo en cuenta los umbrales establecidos oficialmente en el plan Meteoalerta para cada comarca considerada). En general se establecerán distintos intervalos según de favorable sea el entorno a priori, cada uno denotado con un color, en función de diversas reglas o algoritmos. Los ajustes para cada variable han sido probados empíricamente y proporcionan en general unos resultados razonablemente buenos, dentro de la indudable dificultad de proporcionar estimaciones automáticas de zonas con entornos potencialmente adversos. Lógicamente la bondad de la aplicación también reside en gran medida, en la habilidad del modelo en la simulación de las situaciones.

El desarrollo de la página y los productos contenidos se ha llevado a cabo a partir a su vez de la implementación de diversas macros en el sistema McIdas (necesarias para la creación de campos derivados) y de campos ya disponibles o generados en el propio McIdas en el postproceso básico rutinario a partir de macros ya existentes desde hace bastante tiempo (muchas generadas por ATAP/STAP). A su vez ha habido que desarrollar campos citados en la bibliografía internacional (principalmente norteamericana) y adaptar sus rangos de validez a los de España, y a su vez crear parámetros finales de tipo empírico. Finalmente es necesaria una programación en entorno web para organizar y presentar toda la información.

Ciñéndonos estrictamente al diagnóstico convectivo, nos encontramos que aparecen 8 diferentes categorías que a su vez engloban varios campos y parámetros cada una. Son las siguientes: Convección (general), Forzamiento dinámico, Campos e índices termodinámicos, Cizalladura/organización, Granizo y rayos, Supercélulas y tornados, Vientos convectivos no tornádicos y Precipitaciones intensas.

Hay que hacer constar que el mismo parámetro o índice puede formar parte de uno o más bloques debido a su polivalencia. La identificación de los distintos elementos de la página se muestra en la figura 2.
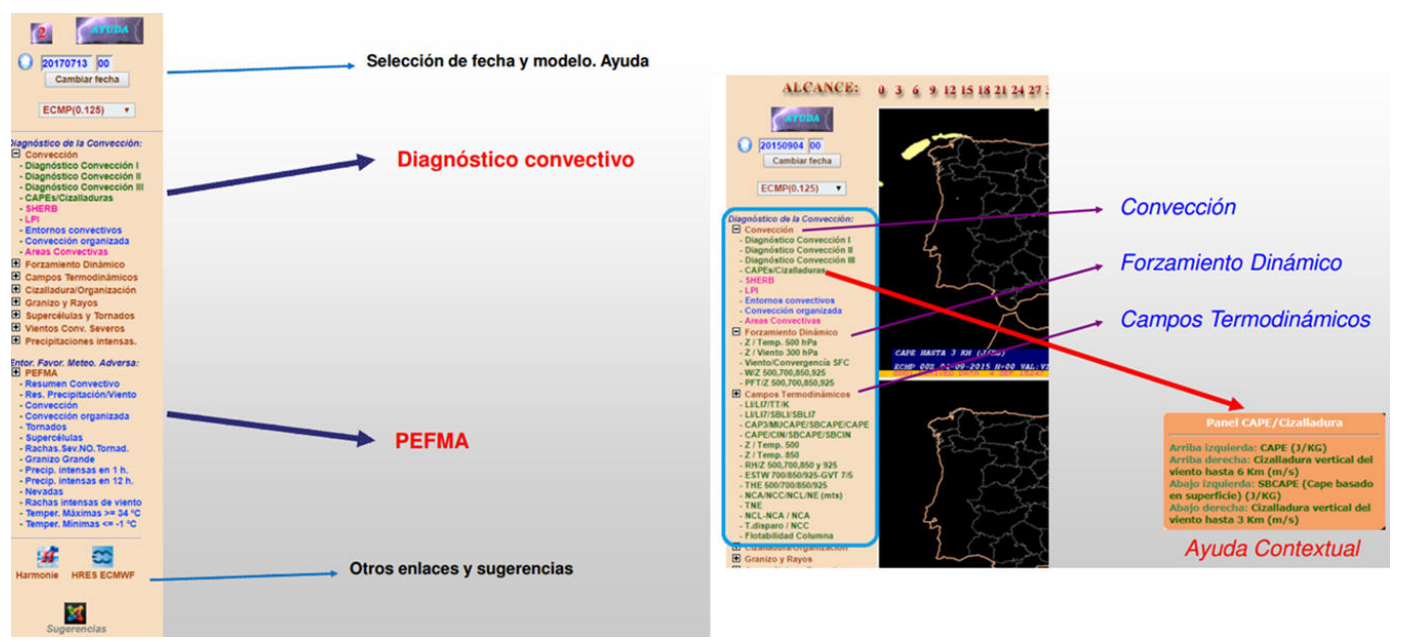

Figura 2. Elementos explicados de la página. Izquierda: Selección de fecha y hora, diagnóstico o PEFMA, y enlaces varios. Derecha: Tipos de productos del diagnóstico convectivo y ayuda contextual.

\section{DIAGNÓSTICO DE LA CONVECCIÓN}

La convección debe entenderse como la existencia de corrientes verticales marcadas, y no ir vinculada a la existencia de tormenta. Para la aparición de la actividad eléctrica han de existir otros factores de carácter microfísico. Por tanto, en general, no podemos asociar exactamente convección con tormenta, y este es un hecho a tener en cuenta en la aplicación.

Además en esta página se aborda el diagnóstico desde un punto de vista de un modelo determinista e hidrostático como es el HRES-IFS del Centro Europeo, donde la convección no está resuelta explícitamente. Es 
por ello que se presentan productos que servirán al predictor para estimar las zonas donde el modelo prevé «indirectamente» que puede desarrollarse la convección si se llega a disparar.

Los errores de interpretación pueden provenir de varios elementos y circunstancias: fallos en las simulaciones del modelo, discontinuidad temporal de 3 horas en la presentación de los productos según cada periodo de predicción que puede hacer obviar estructuras de ciclos de vida cortos, campos en algunos casos excesivamente derivados y aproximaciones no precisas de los algoritmos en determinadas situaciones complejas. Además, en muchos productos se estima el tipo de convección o de fenómeno convectivo asociado, pero finalmente la convección puede no llegar a dispararse. Es por esto que uno de los papeles clave del predictor es determinar con los medios a su alcance, si la convección aparecerá o no, y para ello debe valorar cuantitativamente los ingredientes para dicho disparo, lo cual no siempre es fácil. Por último, se presentan en muchos casos productos finales, habiendo sido ajustados empíricamente, que muestran los entornos favorables (con distintas graduaciones en cuanto a la mayor o menor condición favorable a convección). Luego el predictor tendrá que validar las salidas directas de estos campos finales. También hay que tener en cuenta que los valores de entornos favorables a convección y a determinados fenómenos convectivos están calibrados para el modelo operativo actual HRES-IFS del ECMWF. Por tanto las referencias cuantitativas no deben ser empleadas en otros modelos.

Operativamente se incluyen en este apartado de convección en la herramienta, tres tipos de productos: campos básicos combinados para el diagnóstico convectivo general, índices y parámetros experimentales (LPI, SHERB), y otros que tratan de delimitar áreas que presentan entornos favorables a que pueda desarrollarse convección y el tipo de organización esperada. Se muestran además dos productos que son análogos a los de la antigua página de diagnóstico convectivo del ATAP.

En cuanto a la clasificación de la convección, se han tomado dos características para su cuantificación y categorización: el valor de CAPE (intensidad de la convección) y de la cizalladura vertical del viento (organización), con clasificación por intervalos a partir de valores umbrales obtenidos empíricamente.

- Intensidad de la convección (en función del CAPE):

- Alto CAPE $(\mathrm{CAPE} \geq 500 \mathrm{~J} / \mathrm{kg})$

- Bajo CAPE $(\mathrm{CAPE}<500 \mathrm{~J} / \mathrm{kg})$

- $\quad$ Organización (en función de la cizalladura en los primeros $6 \mathrm{~km}$ ):

- CIZ $6 \geq 10 \mathrm{~m} / \mathrm{s}$

- CIZ6 $<10 \mathrm{~m} / \mathrm{s}$

Las tormentas organizadas intrínsecamente son las que se desarrollan en un ambiente de importante cizalladura vertical del viento. En principio la convección organizada está asociada a tormentas potencialmente severas (SCM, líneas de turbonada, supercélulas, derechos, etc.) Por eso quizás el procedimiento más simple de

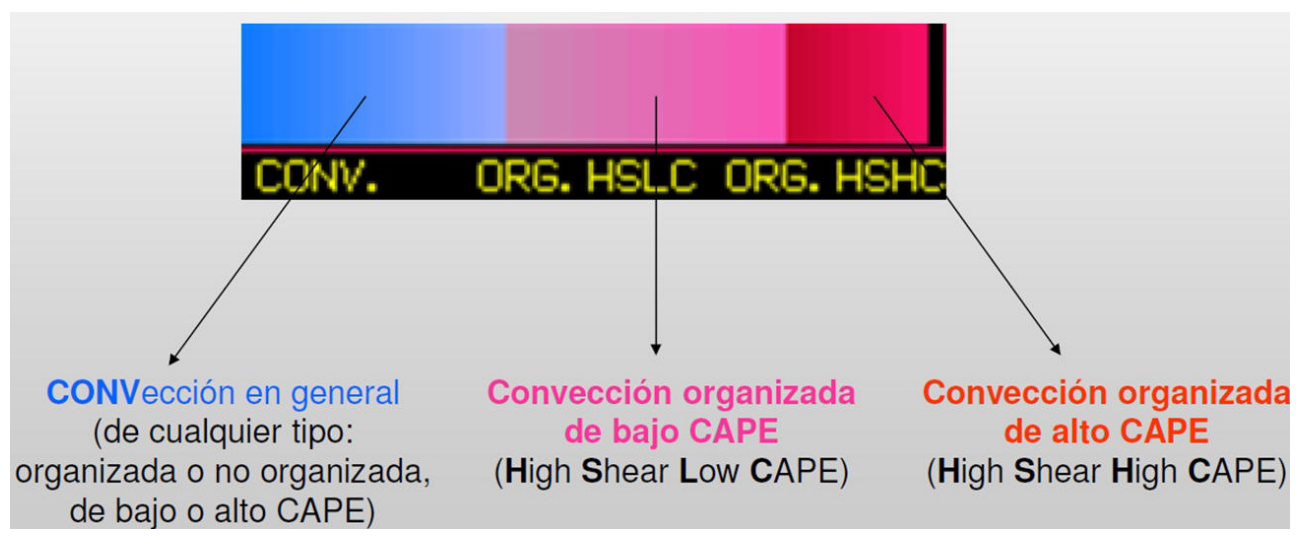

Figura 3. Tipos de convección en función de la Cizalladura (entre 0 y 6 km) y el CAPE. 
clasificación de la convección puede ser en organizada / no organizada en función de si se supera el umbral de $10 \mathrm{~m} / \mathrm{s}$ de cizalladura en los primeros $6 \mathrm{~km}$ en el entorno considerado. También podríamos a su vez clasificar los dos tipos de convección organizada en virtud del valor del CAPE (alto CAPE o bajo CAPE), según se supere o no el umbral de $500 \mathrm{~J} / \mathrm{kg}$. Por tanto dentro de la convección organizada (High Shear) tendríamos dos categorías dependiendo del valor del CAPE: HSLC (High Shear Low CAPE) y HSHC (High Shear High CAPE). Las zonas con entornos favorables a convección en general constituirían otro tipo aparte (figura 3).

\subsection{Entorno favorable a convección}

Se entiende en este caso convección en el sentido de intensas corrientes ascendentes, y no como sinónimo de tormenta eléctrica, cuya generación depende además de otros factores, especialmente de tipo microescalar. De hecho pueden existir, por ejemplo, bastantes casos con troposfera inestable pero «muy fría» (con tropopausa significativamente baja) en la que habrá corrientes verticales intensas sin gran desarrollo vertical y por tanto sin rayos. También en los episodios de lluvias cálidas pueden existir intensas corrientes verticales en un espesor relativamente somero junto a superficie, pero sin rayos. En definitiva este apartado trata de establecer las zonas donde pueden producirse entornos favorables a cualquier tipo de manifestación convectiva (somera o profunda; organizada o no organizada, etc.). La regla empleada (figura 4) tiene en cuenta los índices Lifted Index (LI: hasta 500, y LI7 hasta $700 \mathrm{hPa}$ ) a partir de una capa mezclada de $100 \mathrm{hPa}$ junto a superficie, así como la existencia de valores de CIN iguales o inferiores a $200 \mathrm{~J} / \mathrm{kg}$ y precipitación convectiva prevista por el modelo en las siguientes 3 horas, igual o superior a $1 \mathrm{~mm}$.

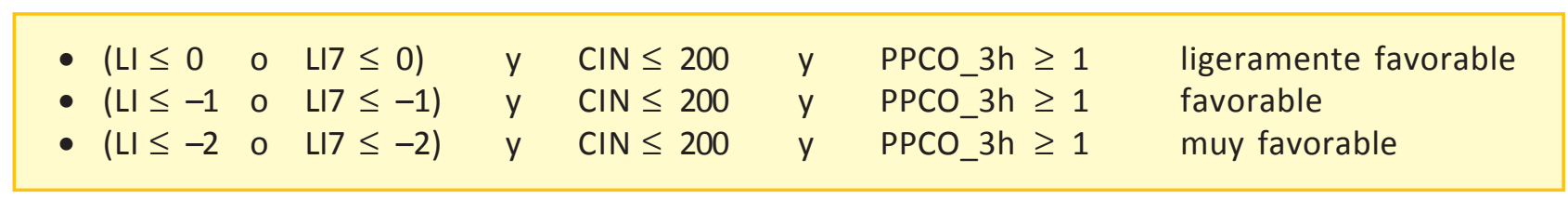

Figura 4. Regla para entornos favorables a convección (ligeramente favorable, favorable o muy favorable).

Hay que tener presente que puede haber ocasiones en que exista convección que finalmente ha sido disparada por diversos mecanismos locales, tales como convergencias mesoescalares, zonas orográficas, fronteras en capas bajas, etc., que no sean bien reproducidas por el modelo, y erróneamente no sean consideradas en algunos casos como áreas convectivas. Por el contrario, en ocasiones puede aparecer como convectiva alguna zona muy reducida y aislada, sin trazabilidad espaciotemporal, que no esté claramente ligada a existencia de movimientos convectivos. Otro elemento importante a tener en cuenta es que en cada paso de tiempo se presenta y tiene en cuenta la precipitación en las próximas horas, por lo que estaríamos considerando la convección en la hora nominal de validez de la predicción y en las siguientes.

En la figura 5 se presenta un ejemplo de salida gráfica de entornos favorables a convección.

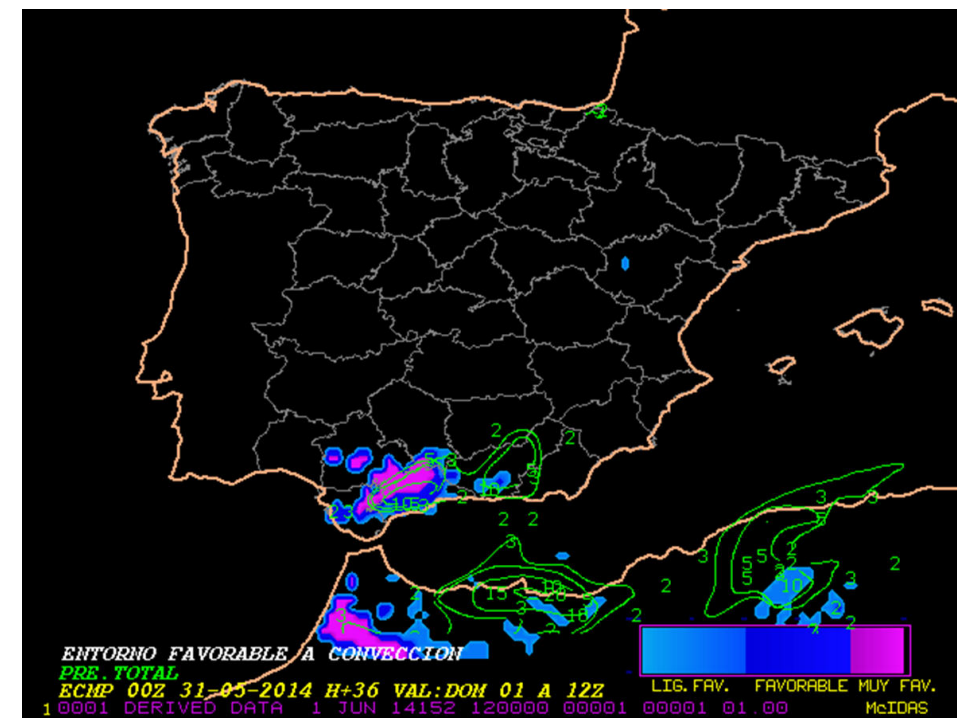

Figura 5. Entornos favorables a convección el 31 de mayo de 2014 a 12 UTC y precipitación total esperada en las siguientes 3 horas. 


\subsection{Entorno favorable a convección organizada}

En este producto se trata de discriminar zonas favorables a entornos convectivos organizados, distinguiéndose tres tipos de entornos convectivos (figura 6): entorno favorable para convección de cualquier tipo (organizada o no organizada, $\boldsymbol{C}$ ), entorno favorable para convección organizada de elevada cizalladura y bajo CAPE (High Shear Low CAPE, HSLC) y entorno favorable para convección organizada de elevada cizalladura y elevado CAPE (High Shear High CAPE, HSHC).

$$
\begin{aligned}
& \text { - }\left[(\mathrm{LI} \leq 0 \text { o } \mathrm{LI7} \leq 0) \quad \text { y } \mathrm{CIN} \leq 200 \text { y } \mathrm{PPCO} \_3 \mathrm{~h} \geq 1\right] \quad \mathrm{C} \\
& \text { - }[(L I \leq 0 \text { o } L I 7 \leq 0) \text { y } K \geq 26 \text { y } C I N \leq 200 \text { y PPCO_3h } \geq 1] \text { y } 0<\text { SBCAPE } \leq 500 \text { y CIZ6 } \geq 10 \text { C. HSLC } \\
& \text { - }[(\mathrm{LI} \leq 0 \text { o } \mathrm{LI} 7 \leq 0) \text { y } \mathrm{K} \geq 26 \text { y } \mathrm{CIN} \leq 200 \text { y } \mathrm{PPCO} 3 \mathrm{~h} \geq 1] \text { y } \mathrm{SBCAPE}>500 \quad \text { y } \mathrm{CIZ6} \geq 10 \text { C. HSHC }
\end{aligned}
$$

Figura 6. Regla para entornos favorables a convección (según la convección se prevea ligeramente favorable, favorable o muy favorable).

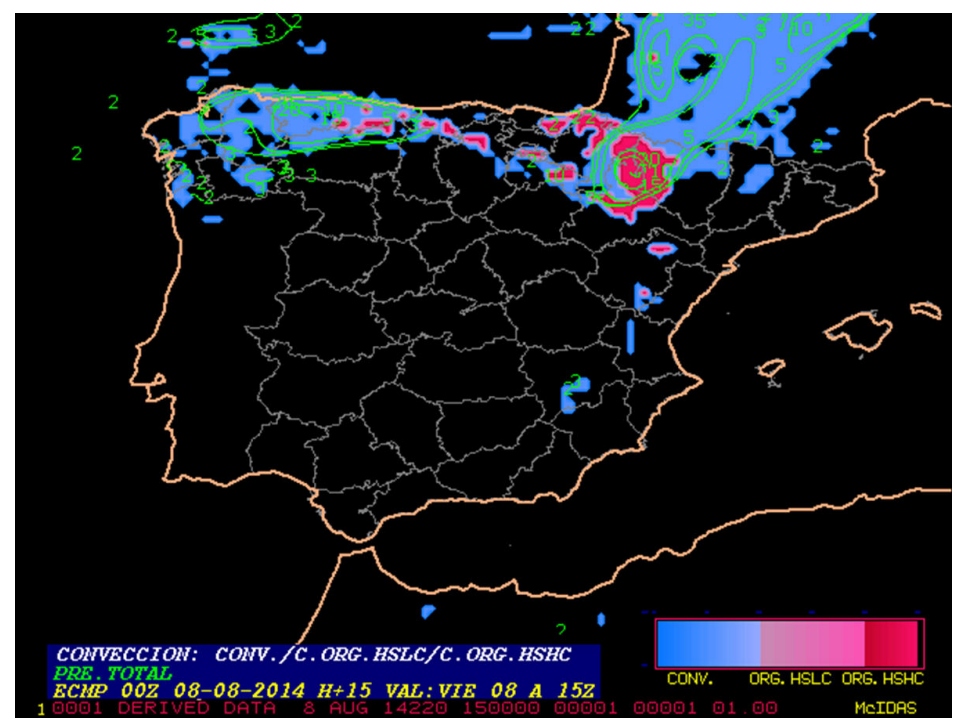

Figura 7. Entornos favorables a convección organizada el 8 de agosto de 2014 a 15 UTC y precipitación total esperada en las siguientes 3 horas. En tonos rosa y rojo se destacan las zonas favorables a convección organizada de bajo y alto CAPE respectivamente.
Se entiende que los entornos de convección organizada son favorables a la aparición de algún tipo de fenómeno adverso en superficie. Como ya se ha comentado, para la discriminación del tipo de convección se utilizan fundamentalmente los índices SBCAPE(Surface Based CAPE, que proporciona el CAPE en una evolución que parte exactamente desde superficie), y CIZ6 (cizalladura vertical del viento en la capa de los 6 primeros kilómetros junto a superficie). El umbral de elevada cizalladura se ha establecido en $10 \mathrm{~s}^{-1}$, y el de elevado CAPE en un valor de SBCAPE de $500 \mathrm{~J} / \mathrm{kg}$. En la figura $7 \mathrm{se}$ muestra un ejemplo de este producto.

\section{CAMPOS Y PARÁMETROS DE FORZAMIENTO DINÁMICO E INESTABILIDAD TERMODINÁMICA}

\subsection{Forzamiento dinámico}

En este apartado de la página web se presentan campos sinópticos básicos y relacionados con la teoría cuasigeostrófica.

- Geopotencial, temperatura y viento en $500 \mathrm{hPa}$.

- Geopotencial y viento en $300 \mathrm{hPa}$.

- Viento y convergencia del viento en superficie.

- Velocidad vertical (W) y geopotencial en $925,850,700$ y $500 \mathrm{hPa}$.

- Parámetro frontal térmico y geopotencial en 925, 850, 700 y $500 \mathrm{hPa}$. 
Los geopotenciales en niveles medios-altos son útiles para determinar forzamiento en altura (ondas) y el aire frío en niveles medios. El parámetro frontal térmico en distintas capas resulta apropiado para identificar la posición de los frentes y además se incluye en el producto presentado el carácter frío o cálido de la masa advectada. Muy importante es la determinación tanto de las velocidades verticales en distintos niveles (salidas directas del modelo) como de las convergencias en superficie estimadas. Es precisamente la determinación de las convergencias en capas bajas un elemento crucial para determinar si finalmente el disparo de la convección va a tener lugar o no, al aportar una energía extra en el impulso vertical de la masa de aire desde superficie. Muchas veces esto supone un reto para el predictor, de vital importancia en el diagnóstico convectivo.

\subsection{Campos e índices termodinámicos}

Bajo este epígrafe se recogen índices y parámetros relacionados con la estructura térmica vertical de la atmósfera:

- Lifted Index calculado en 700 (LI7) y 500 (LI5) hPa, índice TT e índice K.

- Lifted Index en 700 (LI7) y 500 (LI5) con evolución desde condiciones medias de capa mezclada de los 100 primeros hPa y con evolución desde las condiciones de superficie (SBLI500 y SBLI700).

- CAPE / MUCAPE / SBCAPE / CAPE3 [J/kg].

- CAPE / CIN / SBCAPE / SBCIN.

- Z/T 500.

- Z / T 850.

- Humedad relativa y geopotencial en $925,850,700$ y $500 \mathrm{hPa}$.

- Índice de estabilidad estática húmeda (ESTW) calculada en 925, 850 y 700 hPa y gradiente vertical de temperatura calculado en el estrato $700-500 \mathrm{hPa}$.

- Temperatura potencial equivalente y su advección en distintos niveles $(925,850,700$ y $500 \mathrm{hPa})$.

- NCA / NCC / NCL / NE [m].

- NE en unidades de temperatura $\left[{ }^{\circ} \mathrm{C}\right]$.

- NCL - NCA / NCA [m].

- $\mathrm{NCC}[\mathrm{m}]$ y temperatura de $\operatorname{disparo}\left[{ }^{\circ} \mathrm{C}\right]$.

- Flotabilidad en la columna.

\section{CIZALLADURA Y ORGANIZACIÓN}

Se muestran en este apartado, índices y parámetros relacionados principalmente con la cizalladura vertical del viento y la helicidad relativa a la tormenta:

- Cizalladura vertical del viento a 1, 3 y 6 km (CIZ1 / CIZ3 / CIZ6), fundamental para estimar la organización de la convección si esta llega a formarse (figura 8).

- Viento y geopotencial en SFC, 925, 850 y 700 hPa.

- Helicidad relativa a la tormenta de las células divididas calculada por el método de Bunkers a 1 y $3 \mathrm{~km}$ (SRH1 y SRH3 para las células convectivas que se mueven a derecha e izquierda del viento medio).

- Helicidad relativa a la tormenta de las células divididas calculada por el método de Bunkers en la capa efectiva junto con la base y el espesor de la capa efectiva en metros.

- Índice de helicidad-energía (EHI) / Bulk Richardson Number (BRN), que nos pueden dar una idea del tipo de organización e intensidad de la convección.

Quizás uno de los parámetros más destacables sería el de la helicidad relativa a la tormenta $(S R H)$ en la capa efectiva. La introducción del método de la dinámica interna supuso una mejora en el cálculo de $S R H$ al 


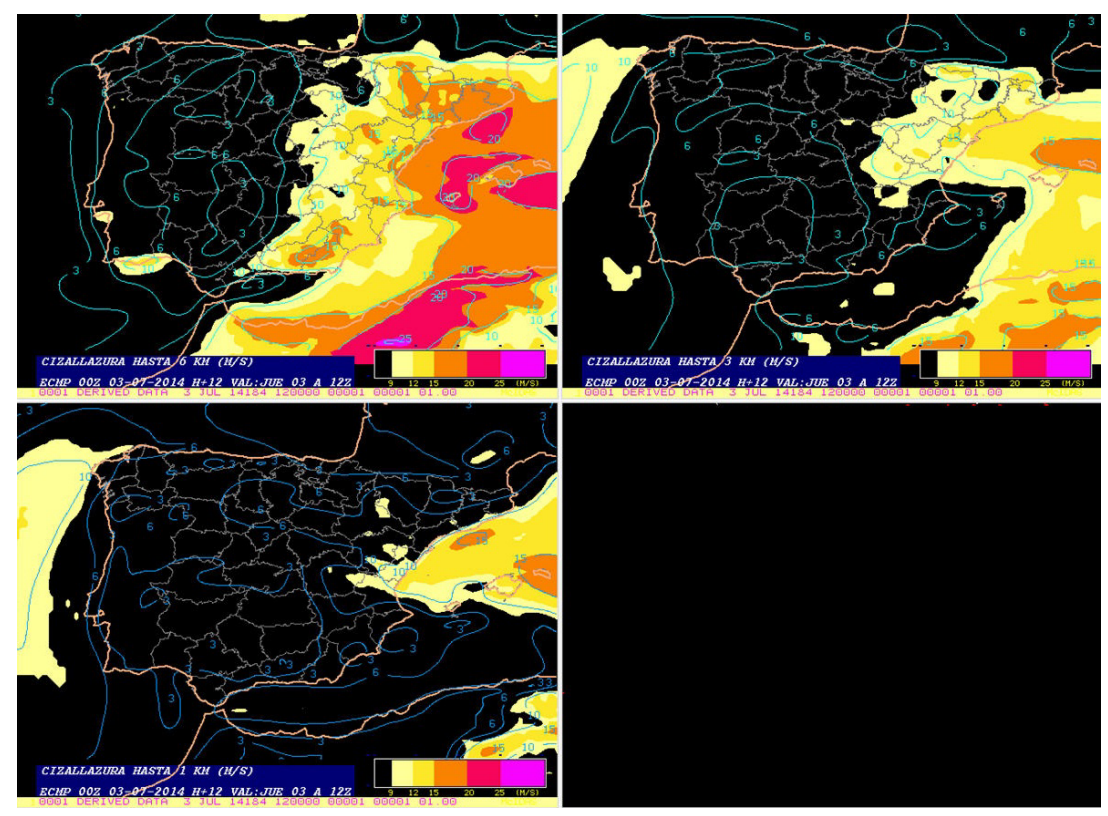

Figura 8. Cizalladura vertical de viento en varias capas desde superficie (1, 3 y 6 km).

mejorar el movimiento estimado de las supercélulas. Con la inclusión del concepto de «capa efectiva» (Thompson et al., 2007), se trata de delimitar el estrato (identificando su base y tope) en el que es más probable que las supercélulas se estén alimentando, y no limitarlo a los estratos inferiores de $1 \mathrm{~km}$ y $3 \mathrm{~km}$ que si bien pueden tener algún fundamento son un tanto arbitrarios. La idea es que no todas las parcelas están en condiciones de formar parte del flujo que alimenta una supercélula. Aquellas parcelas que tienen asociado un alto CIN (energía de inhibición convectiva), o que teniendo un bajo CIN desarrollen poco CAPE, difícilmente van a formar parte del flujo que alimenta una supercélula. Tan solo aquellas con una combinación adecuada de CIN y CAPE lograrán formar parte del flujo alimentador. Esta combinación es la finalmente utilizada. En la ventana propuesta se presenta la helicidad relativa a la tormenta calculada por el método de Bunkers pero

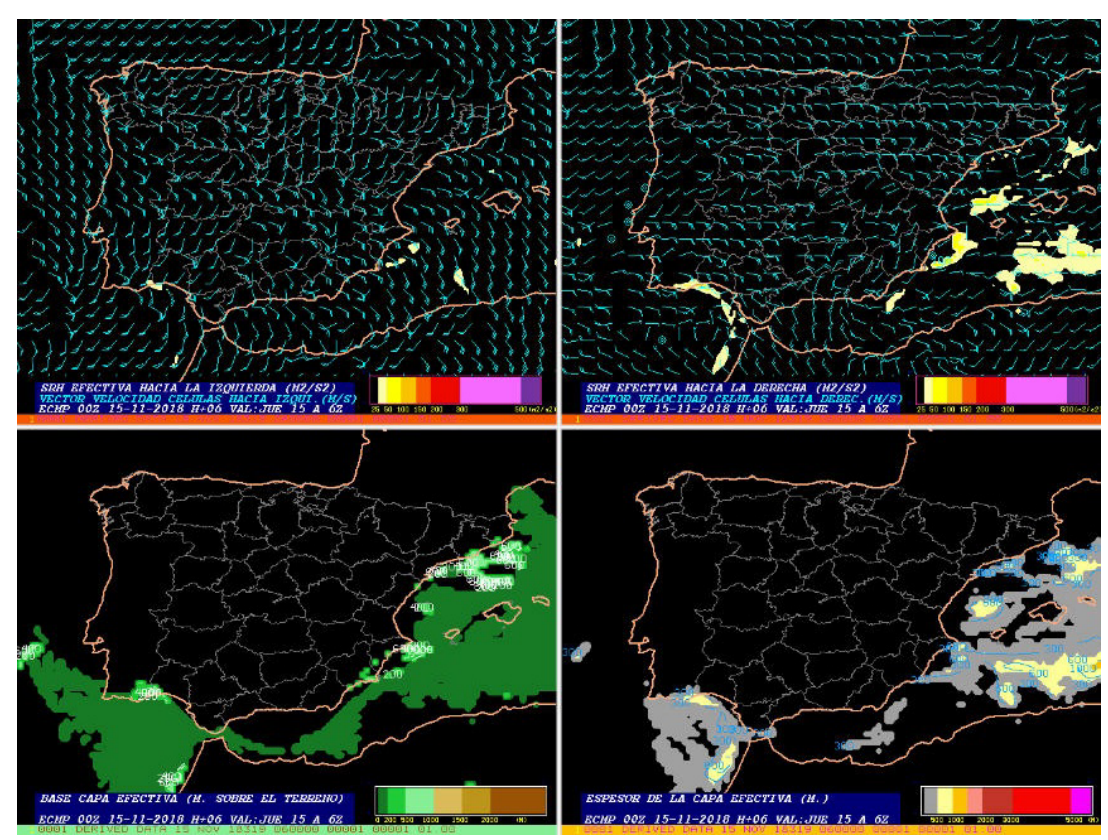

Figura 9. SRH calculada en la capa efectiva $\left(\mathrm{m}^{2} / \mathrm{s}^{2}\right)$. Arriba izquierda: SRH junto con el vector velocidad $(\mathrm{m} / \mathrm{s})$ de las células convectivas que se mueven a la izquierda; arriba derecha: SRH junto con el vector velocidad $(\mathrm{m} / \mathrm{s})$ de las células convectivas que se mueven a la derecha; abajo izquierda: base de la capa efectiva sobre el terreno (m); abajo derecha: espesor de la capa efectiva (m). 
en el estrato definido por la capa efectiva, junto al vector velocidad de las células tormentosas que se mueven a derecha e izquierda ( $V R M$ y $V L M)$, la base y el espesor de la capa efectiva. Este producto suele dar buena señal en casos de tornados y supercélulas (figura 9).

\section{DIAGNÓSTICO DE FENÓMENOS CONVECTIVOS}

También se incluyen en la página, productos y parámetros relacionados con fenómenos convectivos en el sentido de tratar de proporcionar información que permita identificar y cuantificar, a partir de reglas empíricas, los entornos a priori favorables a que se formen dichos fenómenos si realmente la convección se llega a disparar.

Básicamente son índices y parámetros relacionados con granizo grande (figura 10), supercélulas, tornados, vientos convectivos intensos no tornádicos y precipitaciones intensas.

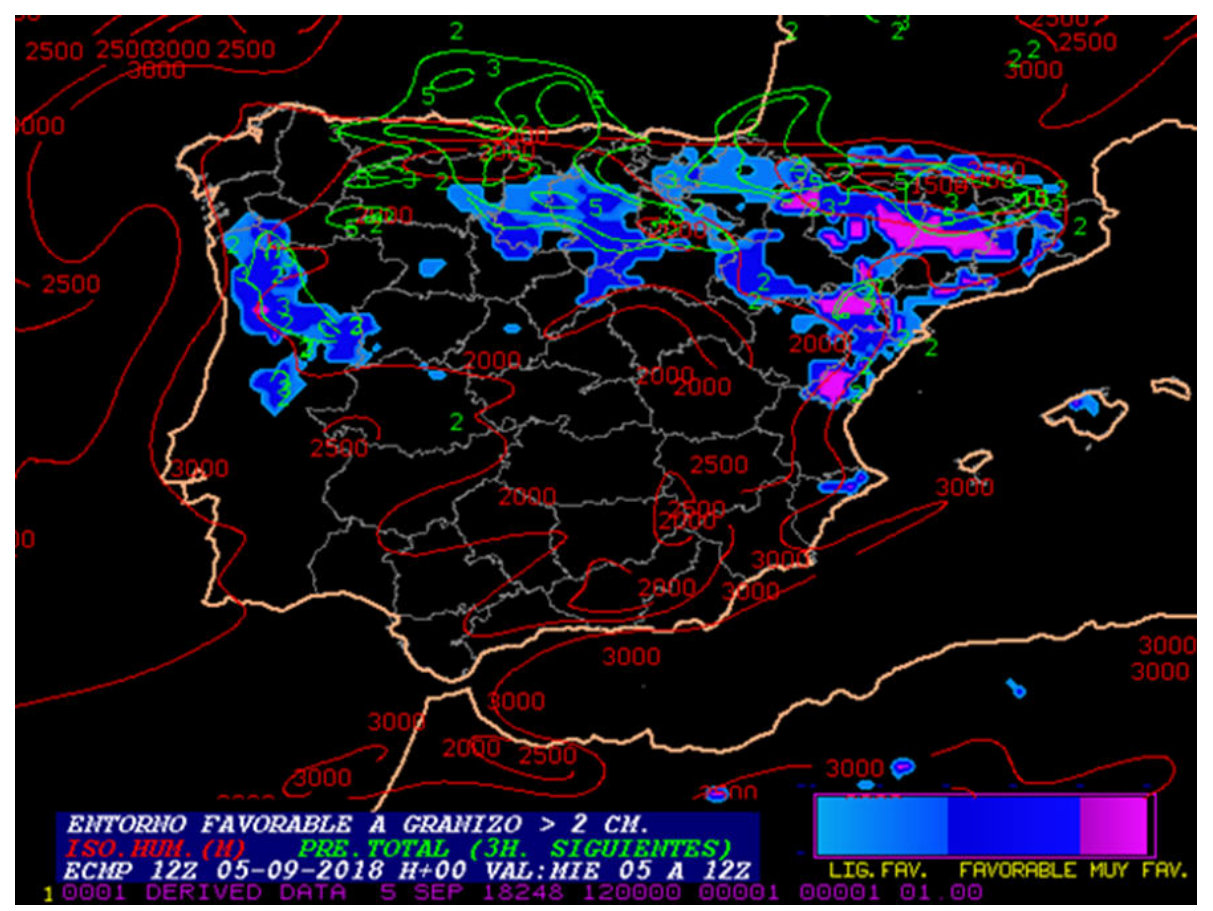

Figura 10. Entornos favorables a granizo grande, el 5 de septiembre de 2018, cuando se recogieron finalmente granizos de más de $7 \mathrm{~cm}$ de diámetro en el NE peninsular. También se incluye la isocero del termómetro húmedo prevista a esa hora y la precipitación total esperada en las siguientes 3 horas.

\section{CONCLUSIONES}

En esencia la herramienta de diagnóstico convectivo está disponible operativamente para el modelo HRES-IFS, tratando de determinar los entornos favorables a la convección y el tipo de convección si esta se llega a disparar. Asimismo muestra espaciotemporalmente los entornos favorables a diversos fenómenos de tipo convectivo. La aplicación puede servir al predictor para la toma de decisiones respecto al pronóstico de la convección y fenomenología asociada. Pero debe ser entendida como una utilidad más de apoyo, a usar no como un producto final sino como un elemento de consulta a tener en cuenta junto con otros productos y herramientas, tales como los derivados de modelos no hidrostáticos como es el caso del modelo operativo Harmonie-Arome. Finalmente la experiencia y conocimientos del predictor en su zona de responsabilidad serán cruciales a la hora de establecer el pronóstico definitivo y la posible emisión de avisos. 


\section{REFERENCIAS}

Craven, J. P. y Brooks, H. E., 2004. Baseline climatology of sounding derived parameters associated with deep moist convection. Nat. Wea. Digest, 28, 13-24.

Griesen, J., 2012. Convection Parameters. http://www.juergen-grieser.de/CovectionParameters/ ConvectionParameters.pdf.

Markowski, P. y Richardson, Y., 2010. Mesoscale Meteorology in Midlatitudes. Wiley-Blackwell. ISBN: 978-0470742136. 430 pp.

Martín León, F., Elizaga Rodríguez, F., Carretero Porris, O. y San Ambrosio Beirán, I., 1999. Curso de Diagnóstico y Predicción de la Convección Profunda, Área de Técnicas de Análisis y Predicción (ATAP). Nota Técnica del ATAPN. ${ }^{\circ}$ 35. AEMET.

Thompson, R. L., Mead, C. M. y Edwards, R., 2007. Effective Storm-Relative Helicity and Bulk Shear in Supercell Thunderstorm Environments. Wea. Forecasting, 22, 102-115.

Sherburn, K. D. y Parker, M. D., 2012. Identifying discriminating environmental features between high shear/low CAPE severe convection and null events. 26th Conference on Severe Local Storms, AMS, 5-8 November 2012, Nashville, TN. 